\title{
ZUR BIOLOGIE DER NIEDERLÄNDISCHEN BRACKWASSERTYPEN
}

\author{
(Ein Beitrag zur regionalen Limnologie)
}

VON

\author{
H. C. REDEKE
}

\section{EINLEITUNG}

Es gibt wohl kaum ein zweites Land, wo das Meer einen so grossen Einflus ausgeübt hat, sowohl auf die Konfiguration des Bodens als auf die Beschaffenheit der Gewässer, wie die Niederlande.

Nicht nur ist der weitaus grösste Teil der westlichen Provinzen ein allerdings durch die Kultur stark veränderter und eingedeichter Haffboden, wo die Dünen noch als Reste der ursprünglichen Nehrung den Abschluss gegen das Meer bilden, sondern auch in den diesen Boden durchfliessenden Wasserstrassen macht sich der Einfluss des Meeres noch heutzutage bemerkbar, insofern als ein grosser Teil dieser mehr oder weniger direkt zusammenhängenden Binnengewässer einen beträchtlichen Salzgehalt aufweist, welcher von einer mittelbaren oder unmittelbaren Vermischung mit Seewasser herrührt.

Sowohl in den unteren Flussgebieten (Aestuarien des Rheins, der Maas und einiger kleinerer Flüsse) wie in vielen von dem Meere abgeschlossenen Binnengewässern Hollands, Zeelands, Frieslands und Groningens ist das Wasser brackisch; im ersten Falle wird dies durch die stetige Vermischung mit bei Flut eindringendem Meerwasser verursacht, im zweiten dadurch, dass namentlich im Sommer, wenn infolge der grösseren Verdunstung in den Kanälen und Gräben das für die Schiffart nötige Wasser fehlt, das Meerwasser durch Schleusen eingeltassen wird, bis der Niveauunterschied ausgeglichen ist.

Hierdurch haben viele Gewässer einen gewissen, oft sehr variabelen Salzgehalt, und auch in den Binnengewässern und namentlich in trockenen Jahren lässt sich der Einfluss des Meerwassers bis auf grosse Entfernung von den Küsten nachweisen.

Die Folge dieser Kontamination mit Meerwasser ist nun, dass die Niederlande reich sind an Brackwassergebieten, welche vom milieu-ökologischen Gesichtspunkt aus ausserordentlich interressant sind. Das Brackwasser ist characterisiert durch eine eigentümliche Fauna und Flora und das Studium der Beziehungen der dort lebenden Gesellschaften zu den Milieufaktoren gehört zu den wichtigsten Aufgaben der Limnologie.

Bei Untersuchungen, welche ich im Laufe der Jahre in etlichen solchen Brackwassergebieten ausgeführt habe, hat sich gezeigt, dass ein eingehendes Studium der Brackwasser-Biocoenosen nicht nur für die Praxis (im Interesse der Fischerei) sondern auch in theoretischer Hinsicht (als Gegenstand der Limnologie) von grosser Bedeutung ist, insofern die in den Brackwassergesellschaften lebenden Arten gewöhnlich nur in geringer Zahl vorhanden sind, die Zahl der. Individuen jedoch meistens sehr gross ist.

Das Leben im Brackwasser ist daher in der Regel sehr üppig, die Gewässer gehören fast sämtlich zu dem polytrophen Typus, sind daher sehr „fruchtbar” und gute Fischgewässer, während 
auf der anderen Seite die verhältnissmässig geringe Zahl der Arten es ermöglichen wird, hier schneller einen Einblick zu bekommen in die gegenseitigen Abhängigkeitsbeziehungen der Organismen und in ihr Verhalten zu den Milieu-Einflüssen, als in dem eigentlichen Süsswasser.

Da nun aber die genaue Kenntniss des Lebensmilieus die wichtigste Voraussetzung der kausalen Limnologie ist, wird es Aufgabe der künftigen Arbeiten sein, neben den mehr vorbereitenden faunistischen und floristischen Untersuchungen zunächst die Eigenschaften des Milieus, i.c. des Brackwassers eingehender $\mathrm{zu}$ untersuchen.

Es hat sich nämlich bei den oben angedeuteten Untersuchungen gezeigt, dass das Brackwasser an und für sich keinen einheitlichen Lebensbezirk darstellt, sondern dass es verschiedene, oekologisch scharf geschiedene Brackwassergebiete gibt, welche ihre charakteristischen Milieufaktoren und dementsprechend ihre eigenen Lebensgesellschaften aufweisen.

Es ist daher vor allen Dingen notwendig, zu einer regionalen Einteilung, einer Systematik dieser Gewässer zu gelangen, welche in ihren chemischen und physikalischen Eigenschaften begründet und nicht nur für rein-theoretische sondern auch für praktische Untersuchungen brauchbar ist.

Durch die vortrefflichen Arbeiten EINAR NAUMANN's 1), welche sich allerdings auf Süsswasser beziehen aber ohne weiteres auch auf Brackwasser ausgedehnt werden können, verfügen wir über ein Prinzip der Einteilung, welches sich auch für unseren Zweck als sehr brauchbar ergeben hat, nämlich die Darstellung des Stoffhaushalts der Gewässer in der Form von sogenannten „Milieu-Spektra." Jedes Spektrum zerfällt in drei grosse Bezirke, „die des übermässig reichlichen Zugangs, die des reichlichen und die des nur spurweisen Vorkommens des betreffen Faktors" (Naumann 1. c. S. 4). Die drei Hauptbezirke werden als poly-, meso- und oligotroph bezeichnet. Das vollständige Fehlen fällt ausserhalb der Spektralbezirke.

Wir sind bis jetzt jedoch noch weit davon entfernt, auch nur ein einigermassen vollständiges Bild des Stoffhaushaltes in unseren Brackwässern geben zu können.

Von den wichtigsten Nährsalzen: Kalziumoxyd, Stickstoff, Phosphorsäure ist noch fast nichts bekannt und es fehlen uns zur Zeit genügende Angaben, um auf Grund des Gehalts an diesen Stoffen eine prinzipielle Einteilung der Brackwässer vor zu nehmen.

Dasselbe gilt für den Gas-Stoffhaushalt. Abgesehen -von vereinzelten $\mathrm{O}_{2}$-Bestimmungen - für den autotrophen Stoffwechsel der Gewässer in erster Linie von Wichtigkeit - liegen auch darüber keine Beobachtungen vor.

Allein über die Temperatur und namentlich über den Cl-Gehalt unserer Gewässer liegt eine grössere Anzahl Daten vor, und da es nur natürlich erscheint, die Einteilung unserer Brackwässer in erster Linie auf Grund des Cl-Gehaltes vorzunehmen, werde ich mich hier zunächst auf diesen Faktor beschränken. Es wird sich zeigen, dass er für unseren Zweck vollkommen ausreicht und dass die vorgenommene Dreiteilung sich im Grossen und Ganzen sehr wohl mit den oekologischen Verhältnissen deckt.

Das Cl-Spektrum für das niederländische Brackwasser dürfte etwa folgende Gestalt haben:

\begin{tabular}{c|c|c} 
oligohalin & mesohalin & polyhalin \\
$0.1-1.0$ gr pro L. & $1.0-10.0$ gr. pro L & über $10.0 \mathrm{gr}$ pro L
\end{tabular}

Hierzu sei bemerkt, dass die Hauptlinien des Spektrums wie ich unten weiter ausführen werde, ziemlich genau den natürlichen Grenzen (soweit man hier von Grenzen sprechen kann) zwischen den Brackwassertypen entsprechen.

Als untere Grenze für "Brackwasser” ist 0.1 gr pro L genommen, weil reines Süsswasser, welches nicht mit Meerwasser kontaminiert ist (Salzseen u. d. bleiben hier ausser Betracht) durchgehends einen $\mathrm{Cl}$-Gehalt von weniger als $100 \mathrm{mg}$ pro L. aufweist. So hat z. B. Regenwasser in Nordholland (Helder) einen mittleren Cl-Gehalt von $33 \mathrm{mg}$ pro L, Wasser aus den Dünen (Trinkwasser par excellence) bis zu 80, Rheinwasser (bei Lekkerkerk und Woudrichem) 54, Vechtwasser (oberer Teil) 42, alles $\mathrm{mg}$ pro $\mathrm{L}$.

1) cf. Naumann, E., Einige Grundlinien der regionalen Limnologie. - Lunds Universitäts Årsskrift. N. F. afd. 2 XVII. No. $8,1921$. 
Das eigentliche Süsswasser bildet somit den Infra-Teil unseres Spektrums: das Chlor spielt hier nur eine untergeordnete Rolle. Auf der anderen Seite geht das Brackwasser unmerklich in das Meerwasser über, letzteres bildet daher gleichsam den Ultra-Teil.

Die Beantwortung der Frage, wie in dieses Spektrum die speziellen Linien, die Abgrenzungen eventueller kleinerer, geschlossener Bezirke eingetragen werden müssen und ob vielleicht auch die Hauptlinien verlegt werden müssen, sobald neben $\mathrm{Cl}$ auch andere Stoffe in Betracht gezogen werden, muss vorläufig dahin gestellt bleiben und späteren Untersuchungen überlassen werden.

Für den Augenblick wollen wir nun die oben aufgestellten Brackwassertypen näher ins Auge fassen und namentlich für jeden die wichtigsten Komponenten der betreffenden Lebensgemeinschaften betrachten. Eine gründliche Bearbeitung des Materials würde jedoch weit über den Rahmen dieser Arbeit. hinausgehen und auch bei dem jetzigen Stand unseres Wissens wohl kaum möglich sein. Namentlich auf botanischem Gebiet stehen hier noch viele unerlässliche Vorarbeiten aus; fehlt uns doch zur Zeit z. B. noch eine monographische Bearbeitung der einheimischen Wasserpflanzen nach oekologischen Grundlagen. Die hier folgende Uebersicht enthält daher nur die wesentlichsten Ergebnisse unserer eigenen Untersuchungen, wird aber hoffentlich auch andere anregen, in der gleichen Richtung weiter zu arbeiten und die noch vorhandenen Lücken allmählich aus zu füllen.

\section{DIE OLIGOHALINEN GEWÄSSER}

Dieser schwach-brackische Typus wird in den Flüssen durch das obere Aestuariengebiet vertreten, im Binnenlande durch eine Anzahl von Gewässern, welche alle ziemlich weit vom Meere entfernt und der Kontamination mit Meerwasser daher nur in geringem Maasse ausgesetzt sind. In Holland und Friesland gehören hierher z. B. das Abcouder Meer mit darin ausmündenden Gewässern sowie der Merwedekanaal, der̈ Amstel, Kager Meer, Brasemer Meer, Zuiderspaarne, Poel bij Amstelveen, Alkmaarder Meer (im Winter und Frühling) und ein grosser Teil der „Friesche Meren”, mitsamt den Poldergewässern im Bereich dieser grösseren Ansammlungen.

Diese Gewässer sind im Allgemeinen dadurch charakterisiert, dass viele Arten aus dem Süsswasser, sowohl Pflanzen wie Tiere, hier noch vorzüglich gedeihen, ja, wie es scheint, manchmal eine Ueppigkeit entfalten, wie sie im reinen Süsswasser selten beobachtet wird.

Unter den submersen Phanerogamen sind die Nymphaeaceen, sowie Limnanthemum und Hydrocharis noch recht häufig, namentlich da, wo infolge der hydrologischen Verhältnisse der Cl-Gehalt im Laufe des Jahres nur wenig schwankt. Im Alkmaarder Meer z. B. fehlen diese Pflanzen vollständig, weil der Cl-Gehalt im Sommer und Herbst zu gross ist. Stratiotes, welche eine rechte Süsswasserart $\mathrm{zu}$ sein scheint, tritt hier nur noch vereinzelt auf.

Plankton. Das oligohaline Phytoplankton ist vor allem durch das massenhafte Auftreten gewisser Cyanophyceen (Microcystis aeruginosa, Oscillatoria agardhi) und Diatomeen (Asterionella gracillima, Thalassiosira baltica var. batava) charakterisiert. Bemerkenswert ist das Vorkommen einiger ChaetocerasArten. so Ch. mülleri im Kager Meer und Ch. diversicurvatum im Abcouder Meer. In den Flussmündungen sind es Coscinodisci, welche bisweilen massenhaft auftreten, z. B. Coscinodiscus rothii im Hollandsch Diep.

Typische Flagellaten sind hier Synura uvella und Eudorina elegans, welche dem Plankton ein ganz eigenes Gepräge verleihen, sowie einige Peridinium-Arten aus der Aciculiferum-Gruppe. Ceratium hirundinella, eine weitverbreitete Art im Süsswasser, wird hier nur noch ganz vereinzelt (eingeschleppt) beobachtet.

Von dem Zooplankton seien hier nur einige Vertreter der wichtigsten Gruppen aufgeführt. Von den Cladoceren ist Leptodora kindti hier sehr zu Hause und wird selbst in kleinen, untiefen Gräben manchmal in grosser Menge gefangen. In den tieferen Gewässern ist Diaphanosoma brachyurum eine häufige Erscheinung.

Die Copepoden sind hier hauptsächlich vertreten durch Diaptomus gracilis, Eurytemora velox (am Ufer) und E. affinis, Cyclops vicinus, $C$. leuckarti und $C$. viridis var. pelagica.

Von den Rotatoren gehören zunächst die auch im Süsswasser vorkommenden Synchaeta-Arten hierher: S. pectinata, S. tremula, S. oblonga. Ferner Asplanchna brightwelli und vor allem Polyarthra platyptera, welche hier bisweilen in unglaublichen Mengen auftritt (Amstelveensche Poel). 
Ufer- und Bodentiere. Das Studium der litoralen und Bodenfauna unserer Gewässer steht noch sehr im Anfang, und mehr noch als beim Plankton muss ich mich hier beschränken auf einige wenige Beispiele.

Eine Hydroide, welche hier sehr verbreitet ist, ist Cordylophora lacustris. Sie tritt namentlich in dem Uebergangsgebiet zu dem mesohalinen Brackwasser auf, führt auf der anderen Seite, im reinen Süsswasser, eine kümmerliche Existenz.

Von den Spongien ist Ephydatia fluviatilis hier noch sehr häufig, Euspongilla scheint ganz auf das süsse Wasser beschränkt zu sein.

Unter den Crustaceen sind die Isopoden hauptsächlich vertreten durch Asellus aquaticus, die Amphipoden durch Gammarus pulex aus dem Süsswasser; Corophium fehlt. Die merkwürdige Brackwasserkrabbe Heteropanope tridentata dringt hier nur an vereinzelten Stellen vor. Dasselbe ist der Fall mit Balanus improvisus; beide haben ihre Hauptverbreitung in dem mesohalinen Bezirk.

Die Mollusken sind grössenteils durch Süsswasserarten vertreten. Dreissensia polymorpha dürfte hier ihre optimalen Existenzbedingungen finden; die seltene Dr. cochleata scheint einen etwas höheren Cl-Gehalt zu bevorzugen.

Auch die aquatilen Insektenlarven sind in diesem Gebiet fast ausschliesslich durch Süsswasserformen vertreten. Soweit mir bekannt ist, fehlen typische oligohaline Arten.

Für die grosse und wichtige Gruppe der Würmer fehlen bis jetzt noch die nötigen Daten.

\section{DIE MESOHALINEN GEWÄSSER}

Ich betrachte dieses Gebiet als das eigentliche Brackwassergebiet s. str. Es wird in den Flüssen durch das mittlere Aestuariengebiet vertreten und in dem Binnenlande durch eine Anzahl von Gewässern in den Meeresprovinzen, von welchen allerdings bis jetzt nur die Gewässer Nordhollands eingehender untersucht wurden. Dieselbe stehen alle mehr oder weniger in Verbindung mit den grossen Kanälen durch welche das Meerwasser nötigenfalls den Binnengewässern zugeführt wird. Es gehören hierher also in erster Linie die beiden Hauptkanäle Nordhollands: „Noordzeekanaal” und "Groot Noordhollandsch Kanaal", sowie zahlreiche andere hiermit offen oder durch Schleusen in Verbindung stehende Wasseransammlungen, z. B. der nördliche Teil des Spaarne, die Seitenkanäle des Nordseekanals, das IJ, das Alkmaarder Meer (im Sommer und Herbst), die Gräben und Kanäle der meisten Polder und die Zijpe. Auch die ganze südliche Zuiderzee, etwa bis zur Linie EnkhuizenStavoren, gehört zu diesem Gebiet.

Diese Gewässer sind hauptsächlich dadurch charakterisiert, dass hier eine Menge von Arten, welche in den beiden anderen Gebieten entweder vollständig fehlen oder nur an der Grenze anzutreffen sind, ihre optimalen Existenzbedingungen finden. Es ist das Gebiet der autochthonen Brackwasserformen und deshalb habe ich es als das Brackwassergebiet s. str. bezeichnet.

Von den submersen Phanerogamen fehlen die Süsswasser-Arten mit wenigen Ausnahmen (Potamogeton pectinatus). Stellenweisse treten sehr häufig Ruppia und Zannichellia auf. Aus dem Wattenmeere dringen Zostera marina sowie etliche Algen in die Zuiderzee bis an die Grenze des oligosảlinen Gebietes vor, jedoch nur in kümmerlichen Exemplaren. Von den Uferplanzen unserer Gewässer geht das Rohr (Phragmites communis) am weitesten hinaus ${ }^{1}$ ).

Plankton. Das mesohaline Phytoplankton führt einige sehr auffallende Diatomeen. In der Zuiderzee herrschen Thalassiosira baltica und Coscinodiscus biconicus vor. Erstere ist auch in den anderen Gewässern dieses Typus häufig, die zweite ist eine autochthone Zuiderzeeform. In den Binnengewässern kommt stellenweise die niedliche Nitzschia acicularis massenhaft zur Entwicklung, Sceletonema costatum tritt hier in einer subtilen Form auf. Sehr charakteristisch für die untere Zone dieses Bezirks ist das bisweilen massenhafte Auftreten verschiedener zarter Chaetoceras-Arten, welche zum Teil in den Amsterdammer Grachten gefunden wurden: Ch. ceratosporum, Ch. crinitum und Ch. wighami. Ch. subtile dagegen ist durch das ganze Gebiete verbreitet.

Von Cyanophyceen finden sich hier namentlich Aphanizomenon flos aquae und Anabaena-Arten, häufig Wasserblüte bildend; sie fehlen jedoch in der Zuiderzee.

1) Es wäre lohnend, die hier an und in den Rohrstengeln und Rhizomen lebenden Tiere, überhaupt die Brackwasserfazies ähnlicher im süssen Wasser verbreiteter Biocönosen eingehender zu untersuchen. 
Unter den Flagellaten ist Ebria tripartata, welche nicht nur in der Zuiderzee, sondern auch in den Binnengewässern auftritt, geradezu als Leitform zu betrachten.

Auffallend ist die Armut an Peridineen und namentlich das vollständige Fehlen von Ceratium-Arten.

Das Zooplankton ist bedeutend ärmer an Arten wie im oligohalinen Bezirk. Die Cladoceren sind vertreten durch Podon polyphemoides - die oligohalinen Arten kommen nur verirrt vor - die Copepoden vor allem durch Acartia bifilosa und Eurytemora hirundoides. Cyclops-Arten fehlen fast vollständig; die obengenannten oligohalinen Species finden sich im Uebergangsbezirk; dagegen tritt in der Zuiderzee die Gattung Halicyclops mit $H$. magniceps auf.

Von den Rotatorien sind namentlich die Synchaeten interessant; es leben z. B. in der Zuiderzee einige Arten, welche gelegentlich auch in den mesohalinen Binnengewässern beobachtet werden, doch anderswo fehlen. Es sind dies hauptsächlich $S$. litoralis und $S$. triophthalma. Hierher gehören auch die typische Brackwasserart Branchionus mülleri und merkwürdige Pedalion fennicum, welche bis jetzt allerdings nur sporadisch beobachtet wurde.

Ufer- und Bodentiere. Cordylophora lacustris (siehe oben) ist in den unteren mesohalinen Zonen noch recht häufig, scheint jedoch zu fehlen, wo der Cl-Gehalt zu hoch wird.

Von Spongien kommen hier und dort nur noch degenerirte Stücke von Ephydatia vor: eigentliche Brackwasserschwämme wurden bis jetzt_nocht nicht gefunden.

Die Würmer sind in diesem Gebiete hauptsächlich durch massenhaft auftretende. Oligochaeten vertreten, sowie durch einige typische Brackwasser-Polychaeten, von welchen hier nur Streblospio dekhuyzeni, Alcmaria romijni und Haplobranchus aestuarinus genannt seien. Aus dem polyhalinen Bezirk dringt Nereis diversicolor am weitesten, bis in die Flussmündungen vor und ist sogar in den Binnengewässern häufig.

Auch unter den Crustaceen ist die Zahl der typischen mesohalinen Arten nicht gering. Asellus, welche hier allerdings noch ab und zu beobachtet wird, ist durch Sphaeroma rugicauda ersetzt, welche bisweilen in grosser Zahl die Rhizome der Uferphanerogamen bewohnt. Zu erwähnen ist hier auch das Vorkommen vori Anthura gracilis, einem häufigen Bewohner der Kanäle in Nordholland und des Unterlaufes der Eem.

Die Amphipoden sind hauptsächlich vertreten durch Gammarus locusta $A$ (die mesohaline Varietät) und die beiden Corophium-Arten: $C$. grossipes und $C$. lacustre, die beide in grossen Mengen auftreten können und zwar die erste auf dem Boden, die zweite am Ufer zwischen Pflanzenwurzeln. Heteropanope ist hier sehr verbreitet, auch Balanus improvisus, die erstere jedoch mehr in der unteren Zone. Sehr typisch für dieses Gebiet ist schliesslich die Brackwassergarnele: Palaemonetes varians.

Charakteristische Mollusken sind hier vor allem die weitverbreiteten degenerirten Formen von Cardium edule, Mya arenaria und Macoma baltica, welche in den meisten bisher untersuchten mesohalinen Binnengewässern und namentlich in der Zuiderzee massenhaft vorkommen. Als typische mesohaline Art ist auch Hydrobia jenkinsi zu nennen. Von den Süsswasser- resp. oligohalinen Schnecken ist Neritina fluviatilis noch weit verbreitet; die Najaden sowie die gewöhnlichen Schnecken (Vivipara, Lymnaea u. A.) sind selten oder fehlen ganz.

Die Insekten-Larven sind hier durch ziemlich viele Arten vertreten, welche die Grenze des Bezirks noch gerade $\mathrm{zu}$ überschreiten vermögen; sie halten sich vorwiegend in der unteren Zone auf. Beispiele sind 1. Odonata: Agrion pulchellum, Enallagma cyathigerum, Erythromma naias, Ischnura elegans. 2. Ephemeriden: Caenis halterata und namentlich Cloeon praetextum. 3. Neuroptera: Sialis flavilatera, stellenweise massenhaft. 4. Trichoptera: Anabolia nervosa, Cyrnus flavidus, Limnophilus lunatus und rhombicus. Sämmtliche Species können jedoch nur einen verhältnissmässig geringen ClGehalt vertragen; sobald der Salzgehalt des Wassers zu hoch wird, gehen sie ein. $\mathrm{Ob}$ bei uns tatsächlich echte Brackwasserformen unter den Insekten vorkommen, scheint mir zweifelhaft ${ }^{1}$ ).

\section{DIE POLYHALINEN GEWÄSSER}

Der hochbrackische Typus wird in den Flüssen durch das untere Aestuariengebiet, welches in offener Verbindung mit dem Meere steht, vertreten. Auch der nördliche Teil der Zuiderzee, sowie die

1) Vielleicht gibt es solche unter den sehr verbreiteten Chironomiden. Eine Bearbeitung der im Brackwasser lebenden Arten dieser Gruppe scheint sehr erwünscht. 
in offener Verbindung mit dem Meere stehenden Häfen gehören hierher. Im Binnenlande sind es diejenigen Wasseransammlungen welche in unmittelbarer Nähe der Schleusen gelegen sind. In den übrigen Binnengewässer steigt der Cl-Gehalt nur in ausnahmsweise trockenen Jahren $(1911,1921)$ bis zur Polyhalinität.

Von Phanerogamen wachsen hier die beiden Zostera-Arten: Z. marina und Z. nana, sowie eine grosse Zahl höherer Algen.

Plankton. Das Plankton ist stark beeinflusst durch das Meer: in der nördlichen Zuiderzee kommen viele Arten aus dem Wattenmeer bis an die Grenze der mesohalinen Zone vor. Cyanophyceen fehlen. Von Diatomeen sind Biddulphia aurita und Lithodesmium undulatum sehr charakteristische Arten. Letztere wurde gelegentlich auch in Binnengewässern beobachtet. Der häufigste Coscinodiscus ist hier C. grani.

Peridineen sind auch hier noch selten und ausschliesslich durch aus der Nordsee eingeführte Arten vertreten. Am weitesten dringt Ceratium fusus vor.

Noctiluca miliaris hat hier die untere Grenze ihrer Verbreitung; dasselbe gilt für die zahlreichen Coelenteraten, so für Pleurobrachia pileus. Dagegen hat die eigentümliche, in Holland bisher nur in der nördlichen Zuiderzee beobachtete Nemopsis bachei ihre Hauptverbreitung in dem unteren polyhalinen Bezirk.

In dem Zooplankton fehlen die Rotatoren und Cladoceren fast vollständig. Letztere sind vertreten durch Podon polyphemoides, welche hier ihre obere Verbreitungsgrenze findet. Dagegen sind die Copepoden mit vielen Arten recht zahlreich. So finden sich in der nördlichen Zuiderzee u. A. Acartia discaudata, Centropages hamatus, Euterpina acutifrons, Oithona nana, Temora longicornis. Auch von diesen Arten sind mehrere gelegentlich in Binnengewässern beobachtet.

Ufer- und Bodentiere. Wie beim Plankton so ist besonders auch in dieser Gruppe der Einfluss des Meeres sehr gross, namentlich in der nördlichen Zuiderzee, wo sich eine artenreiche Fauna entwickelt hat, welche in ihren Hauptzügen der des Wattenmeeres gleicht und kaum autochthone Arten enthalten dürfte. Vielleicht lassen sich in den bis jetzt noch weniger untersuchten Tiergruppen solche typische Hochbrackwasserformen auffinden. Uebrigens gehört dieser Bezirk eigentlich schon mehr zu den Objekten der Meeresforschung als zur Limnologie.

Es war in diesem Aufsatz von Fischen nicht die Rede. Ich habe es absichtlich nachgelassen, diese Wasserbewohner in meine Betrachtungen hineinzuziehen, weil es bei den Fischen unserer Fauna meiner Ansicht nach keine typischen Brackwasserarten gibt. Das Anpassungsvermögen der Fische ist so gross, dass sie sämtlich ohne Schwierigkeiten von dem einen Brackwasserbezirk in den andern, oder von dem Süsswasser in das oligohaline Brackwasser oder von dem Meere in das Brackwasser oder sogar in das Süsswasser einwandern können. Nur der Stint, welcher sich gerne in dem mesohalinen Bezirk aufhält, zur Laichzeit aber bis in das reine Süsswasser vordringt, könnte allenfalls als Beispiel eines Brackwasserfisches aufgeführt werden. Aber auch dieser Fisch kann uns bei der biologischen Einteilung unserer Brackwässer keine erheblichen Dienste leisten.

\section{S C H L USS}

Ueberblicken wir die oben mitgeteilten Befunde, so können wir, wie mir scheint, zunächst feststellen, dass die hier vorgeschlagene Einteilung unseres Brackwassers der Hauptsache nach den natürlichen Verhältnissen durchaus entspricht. Obgleich es in der Natur keine Grenzen gibt und, wie schon eingangs erwähnt wurde, die Lage der Linien unseres Cl-Spektrums vielleicht noch nicht als endgültig betrachtet werden darf, so halte ich sie doch im Wesentlichen für richtig, weil es sich gezeigt hat, dass die drei Bezirke eigene und von den anderen verschiedene Lebensgemeinschaften aufweisen.

Es ist einleuchtend, dass diese Gesellschaften innerhalb des Bezirks örtlich sehr verschieden zusammengesetzt sind, dass z. B. die litoralen Gesellschaften im oligohalinen Gebiet andere sind als die profundalen oder pelagischen, aber durchweg findet man in ähnlichen Biocoenosen innerhalb eines bestimmten Bezirks die nämlichen Organismen; sei es auch oft in wechselndem Verhältnis. Das braucht hier nicht näher erörtert zu werden; nur möchte ich noch auf einige Erscheinungen allgemeiner Natur hinweisen. 
$\mathrm{Da}$ ist nun zunächst bemerkenswert, dass mehrere Gattungen in den drei Brackwassertypen durch verwandte, häufig massenhaft auftretende Arten oder Varietäten vertreten sind. Beispiele solcher Triaden sind:

Oligohalinophil

Coscinodiscus rothi

Thalassiosira baltica batava

Chaetoceras mülleri

Eurytemora affinis

Gammarus pulex
Mesohalinophil

Coscinodiscus biconicus

Thalassiosira baltica

Chaetoceras subtile

Eurytemora hirundoides

Acartia bifilosa

Gammarus locusta A

Corophium lacustre und grossipes
Polyhalinophil

Coscinodiscus grani

Thalassiosira excentrica

Chaetoceras debile

Eurytemora hirundo ${ }^{1}$ )

Acartia discaudata

Gammarus locusta

Corophitum crassicorne

Häufig finden sich auch Vertreter verschiedener aber nahe verwandter Gattungen als Hauptrepräsentanten der einzelnen Bezirke. Als Beispiele führe ich hier die Cyclops-Arten an, welche fast ausschliesslich oligohalinophil sind und in dem mesohalinen Gebiet durch Halicyclops, im polyhalinen durch Oithona vertreten sind. Aehnliches beobachten wir bei den planktonischen Cyanophyceen, welche allerdings im Hochbrackwasser fehlen und bei den Polychäten und Corophien, welche umgekehrt im Oligohalinikum fehlen.

Sehr bemerkenswert und für die weitere Brackwasserforschung von Bedeutung scheint mir schliesslich, dass in dem eigentlichen. Brackwassergebiet (im Mesotyp) die Repräsentanten von Gattungen fehlen, welche in den beiden anderen resp. im Süsswasser oder im Meer durch eine oder mehrere Arten vertreten sind. Dies ist z. B. ganz auffallend in der Gattung Rhizosolenia, wo R. longiseta im süssen aber kaum noch im oligohalinen Wasser lebt, während die Gattung im mesohalinen vollständig fehlt und erst in Rh. calcar avis im polyhalinen Gebiet wieder auftritt. Ebenso fehlen in dem eigentlichen Brackwasser Ceratium-Arten, obgleich $C$. hirundinella im reinen Süsswasser sehr häufig ist und die neritische $C$. fusus tief ins polyhaline Gebiet durchdringt. Auch echte Brackwasserschwämme scheinen zu fehlen und von der Gattung Chaetoceras welche sowohl im schwachbrackischen Wasser wie im Meere durch zahlreiche Arten vertreten ist, kommt im eigentlichen Brackwasser bei uns nur eine Art, Ch. subtile, häufig vor.

Es ist schwer einzusehen, weshalb die Lebensbedingungen für die betreffenden Arten hier weniger günstig sind als im Süsswasser oder im Meer, und es wird, wie denn überhaupt bei den vielen Fragen, welche sich an das Studium der Brackwasser-Oekologie knüpfen, Sache zukünftiger Forschung sein, hier die kausalen Beziehungen klar zu legen. Vor der Hand war es meine Absicht, einige dieser Probleme nur ganz kurz an zu deuten, gleichzeitig aber einige Klarheit zu bringen in den bisher noch ziemlich verworrenen Begriff: Brackwasser.

1) Bei uns noch nicht beobachtet. 